\title{
Proper motion and densification of the International Celestial Reference Frame in the direction of the Galactic bulge ${ }^{\star}, \star \star$
}

\author{
R. Teixeira ${ }^{1,2}$, P. A. B. Galli ${ }^{1}$, P. Benevides-Soares ${ }^{1}$, J. F. Le Campion ${ }^{2}$, M. Fidêncio ${ }^{1}$, \\ A. Krone-Martins ${ }^{1,3}$, C. Ducourant ${ }^{2,1}$, and D. Sinachopoulos ${ }^{1,4}$ \\ ${ }^{1}$ Instituto de Astronomia, Geofísica e Ciências Atmosféricas, Universidade de São Paulo, Rua do Matão, 1226 - Cidade Universitária, \\ 05508-900 São Paulo - SP, Brazil \\ e-mail: teixeira@astro.iag.usp.br \\ 2 Observatoire Aquitaine des Sciences de l'Univers, CNRS-UMR 5804, BP 89, 33270 Floirac, France \\ 3 Faculdade de Ciências, Universidade de Lisboa, Ed. C8, Campo Grande, 1749-016 Lisboa, Portugal \\ ${ }^{4}$ Institute of Astronomy and Astrophysics, National Observatory of Athens, I. Metaxa \& Bas. Pavlou, 15236 Palea Penteli, Greece
}

Received 7 July 2011 / Accepted 30 August 2011

\begin{abstract}
Context. The definition, construction and realization of a reference system is one of the oldest and most fundamental tasks of astronomy. Currently, the International Celestial Reference System (ICRS), realized by the International Celestial Reference Frame (ICRF) or ICRF2, is the reference system adopted by the IAU. It is based on the very precise VLBI positions of a few hundred compact extragalactic radio sources. Despite its excellent precision, the ICRF is far from providing a reference system that is available for the entire sky and accessible to all observers. The ICRF has to be densified and extended to other wavelengths, mainly to the optical domain where the astronomical activities are more intense. For this reason, the IAU has recommended and encouraged works in this direction over the years. Many of them were developed to give sometimes good positions and proper motions, but they are very limited in magnitude, while others are extremely dense and deep in magnitude but have low accuracy, mainly for the proper motions. Nevertheless, all these contributions are very important because they are complementary.

Aims. We present a homogeneous and precise optical astrometric catalog that extends the ICRF in the direction of 12 low-extinction windows of the Galactic bulge and provides at the same time a useful database for kinematic studies.

Methods. The catalog was constructed on a total of 9 observational epochs spread over 15 years of observations with the CCD meridian circle of the Abrahão de Moraes Observatory (Valinhos - Brazil). The Galactic Bulge Valinhos Observatory Catalog (GBVOC) contains only those stars with at least 6 observational epochs and a time base of 10 years or more. We also imposed a minimum of 3 observations per star in each observational epoch and 20 observations in total.

Results. The catalog provides positions and proper motions for about 11600 stars with a mean internal precision of about 22 mas and $4.0 \mathrm{mas} / \mathrm{yr}$, which ensures a high quality and reliable extension of the ICRF in this direction. The GBVOC is extremely dense with more than 3500 stars per square degree. Comparison of the GBVOC with other densification works allows us to estimate the external precision of the GBVOC to be better than 42.0 mas and $5.5 \mathrm{mas} / \mathrm{yr}$ in position and proper motion.
\end{abstract}

Key words. Galaxy: bulge - astrometry - reference systems - dust, extinction - catalogs - proper motions

\section{Introduction}

The definition, construction, and realization of a reference system is the first step to study the positions and motions of any body. As in its beginning astronomy focused on the positions and motions of the celestial bodies, we can say that the realization of a reference system is one of the oldest and most fundamental task in astronomy.

The practical problems in realizing a reference system arise from the need to obtain at any given instant the coordinates of any celestial body as accurately as possible in an inertial reference system. This need requires that the adopted reference system (conventional reference system) be accessible, available, homogeneous, and rigid. In other words, it must be realized by a

* Based on observations collected with the CCD meridian circle of the Abrahão de Moraes Observatory - Valinhos - Brazil.

$\star \star$ Full Table 2 is only available at the CDS via anonymous ftp to cdsarc.u-strasbg.fr $(130.79 .128 .5)$ or via

http://cdsarc.u-strasbg.fr/viz-bin/qcat?]/A+A/534/A91 very homogeneous, accurate, dense, and multiwavelength cata$\log$. This implies having a catalog with very accurate positions of a very large number of extragalactic objects or very accurate positions and proper motions of a large number of stars in the case of a stellar-based reference frame. Over time, in the past two centuries, this issue was somewhat solved by a series of fundamental catalogs (Fricke 1985) where the successor represented an extension and improvement of the predecessor.

In 1991 the IAU adopted a new reference system, the International Celestial Reference System (ICRS; Arias et al. 1995; Feissel \& Mignard 1998) realized by the very accurate VLBI positions of extragalactic radio sources in the International Celestial Reference Frame (ICRF; Ma et al. 1998). They satisfactorily solve some of these aspects such as the inertially for example. On this occasion, the IAU ended the tradition of fundamental catalogs and reference systems based on solar system dynamics and introduced a new strategy that was more coherent 
with the current observational capabilities and needs of astronomical research (see for example, Capitaine et al. 2000; Seidelmann \& Kovalevsky 2002). The extremely low density of ICRF or ICRF2 (Fey et al. 2010) and also the fact that it is based on radio positions while most of the astronomical works are done in the optical domain, implies that this reference system must be extended to this domain and densified by optical stellarbased catalogs. The density of the ICRF2 is less than 1 source (defining source or not) by $10^{\circ} \times 10^{\circ}$.

The primary optical realization of the ICRS is ensured by the Hipparcos catalog (ESA 1997), whose astrometric parameters were determined from spatial observations and, until now, gives the best astrometric parameters of all catalogs. The Hipparcos catalog is very limited for magnitudes, essentially brighter than $V \simeq 10$, and it is not as dense since it contains only 3 stars by $1^{\circ} \times 1^{\circ}$, as would be necessary for astronomical research. Therefore some others optical extensions of the ICRF were built to satisfy the requirements cited before: SPM (Platais et al. 1998), Tycho2 (Hög et al. 2000), UCAC2 (Zacharias et al. 2004), PM2000 (Ducourant et al. 2006), PPMX (Röser et al. 2008), UCAC3 (Zacharias et al. 2010), PPMXL (Röser et al. 2010), and UCAC4 (Zacharias 2011, priv. comm.) and many more. On average we can say that we have a mean precision better than $100 \mathrm{mas}$ in postion and $10 \mathrm{mas} / \mathrm{yr}$ in proper motion for these catalogs.

Of course, all these astrometric catalogs play an important role in the realization of the ICRS in the optical domain, but none fully meets the requirements such as quality, accessibility, availability, rigidity, etc. Moreover, in some cases, even if their overall quality is good, punctual proper motion works may be compromised by inaccuracy or large errors.

In this work we present an accurate and homogeneous catalog that aims to extends and densify the ICRF in the optical domain in a limited but very important region of the sky, the direction of 12 low-extinction windows of the Galactic bulge (Blanco 1988; Blanco \& Terndrup 1989; Dominici et al. 1999). This Galactic Bulge Valinhos Observatory Catalog (GBVOC) is based exclusively on CCD meridian circle observations at the Abrahão de Moraes Observatory, Valinhos - Brazil (Viateau et al. 1999). The GBVOC contains a total of 11619 stars with a very rich observational history, ensuring a very high quality for its positions and proper motions. With this catalog, more than the realization of the ICRS in the optical domain, we also aim at its reliable utilization in works such as determination of memberships and stellar kinematics.

The regions considered here are important because they are the only possible access to the Galactic bulge in the optical domain. The bulge is difficult to access because of the large amount of dust and gas, hence the high extinction, so the lower extinction windows, such as Baade's window (Baade 1951) and others found in Blanco (1988) and Blanco \& Terndrup (1989) offer privileged insight into the Galactic bulge (Dominici et al. 1999).

The observational material used in the construction of this catalog is presented in Sect. 2. In Sect. 3 we present the treatment of the observations. In Sect. 4 we present the catalog. In Sect. 5 we compare our results in position and proper motion with those from some of the most important astrometric catalogs. Finally, Sect. 6 discusses our results and draws a conclusion about our work.

\section{Observations}

All observations used here to compose this catalog were realized over 15 years (1996-2010, mean epoch 2002.2), with a single instrument, the Valinhos CCD meridian circle (Viateau et al. 1999; Dominici et al. 1999; Teixeira et al. 2000). This instrument is installed at the Abrahão de Moraes Observatory in Valinhos Brazil $\left(\phi=-23^{\circ} 00^{\prime} 06^{\prime \prime}, \lambda=+46^{\circ} 58^{\prime} 03^{\prime \prime}\right)$ and was automatized in 1995 with the introduction of a CCD micrometer (Viateau et al. 1999).

The CCD meridian circle works in drift scan mode, observing a field with a defined size in declination (14') and an arbitrary length in right ascension. In general, but not always and not for all low-extinction windows, we observed $20 \mathrm{~min}$ in right ascension for each field ensuring enough reference stars (Tycho-2) for the reduction. During the observation, the telescope was fixed and the electric charges moved along the columns of the CCD with the same velocity as the transit.

The diameter of the objective of the telescope is $0.19 \mathrm{~m}$ and its focal distance is $2.6 \mathrm{~m}$ resulting in a scale of 1 pixel $=1.5^{\prime \prime}$ for squares pixels of $19 \mu$. The Thomson 7895A CCD has a matrix of $512 \times 512$ pixels cooled down to $-40{ }^{\circ} \mathrm{C}$. The observations were carried out with a filter CG495+BG38 defining a bandpass of 5200 to $6800 \AA$ (Dominici et al. 1999; Teixeira et al. 2000). Even if this filter does not exactly match the Johnson $V$ one, we use the letter $V$ because it is also in the visual band, i.e., the letter $V$ does not indicate the filter but only the band. The exposure time is $51^{\mathrm{s}} / \cos (\delta)$.

The observational program was started in 1996 and finished in 2010. Unfortunately, in this period, some technical problems (2000 to 2005) meant we were able to observe only nine years, so they constituted a maximum of nine observational epochs to calculate the proper motions.

This program received an important stimulus in the 24th General Assembly of the IAU Manchester in 2000, in a "Densification of reference frame" meeting proposed by Dafydd Wyn Evans and also by the IAU Working Group, "Astrometry by small ground-based telescopes" led by William Thuillot.

In the total we observed more than one hundred thousand stars. However, the catalog contains only the stars that passed by the filters presented in the Sect. 4: minimum number of observation by star and minimum number of observational epoch.

\section{Data reduction}

For each low-extinction window, data were processed using the Bordeaux reduction pipeline (Ducourant et al. 2006), which takes the following steps. First, each observing strip is measured by fitting a two-dimensional Gaussian to each detected object, which leads to a catalog of $(x, y$, flux). Then the measurements are preliminarily reduced classically using the Tycho- 2 catalog as reference to produce a catalog of $(\alpha, \delta, \mathrm{mag})$ and associated errors for each detected object of each strip.

The third step corresponds to the global fit of all observations of the considered window, which allows estimating the stellar parameters of all detected sources (Eichhorn 1960; Ducourant \& Rapaport 1991; Benevides-Soares \& Teixeira 1992; Teixeira et al. 1998). This process is iterative and the convergence is reached after two or three iterations. During this process, observations of a star are weighted with the mean residual of the strips where the star has been measured. During the global fit, a constraint is applied to Tycho- 2 stars so that no drift in the origin of the system is allowed. Finally a catalog of positions and proper motions is derived that contains stellar parameters and associated errors for each object. 
Table 1. Coordinates and statistic properties for each of 12 lowextinction windows in the Galactic bulge direction.

\begin{tabular}{|c|c|c|c|c|c|c|c|}
\hline id & $\begin{array}{l}\text { RA } \\
(\mathrm{hm})\end{array}$ & $\begin{array}{l}\text { Dec } \\
\left({ }^{\circ} \prime\right)\end{array}$ & Stars & $\sigma_{\delta}$ & $\sigma_{\alpha}$ & $\begin{array}{l}\sigma_{\mu_{\alpha} \cos \delta}(\mathrm{mas} / \mathrm{yr}\end{array}$ & $\sigma_{\mu_{\delta}}$ \\
\hline $\mathrm{BE}$ & 1810 & -3145 & 1169 & 21.1 & 23.7 & 3.9 & 4.4 \\
\hline BG & 1818 & -3251 & 1259 & 21.4 & 22.7 & 4.0 & 4.2 \\
\hline BJ & 1840 & -3449 & 524 & 16.7 & 18.2 & 3.2 & 3.4 \\
\hline LA & 1656 & -5244 & 1378 & 22.5 & 23.5 & 4.2 & 4.4 \\
\hline LB & 1718 & -4805 & 842 & 20.7 & 22.0 & 3.9 & 4.2 \\
\hline LC & 1729 & -4520 & 1256 & 22.1 & 22.9 & 4.1 & 4.2 \\
\hline LD & 1744 & -4041 & 980 & 18.9 & 21.1 & 3.6 & 4.0 \\
\hline LI & 1804 & -3347 & 987 & 24.2 & 26.4 & 4.6 & 5.1 \\
\hline LR & 1823 & -2610 & 892 & 21.5 & 24.0 & 4.0 & 4.4 \\
\hline LT & 1834 & -2123 & 907 & 24.9 & 26.9 & 4.2 & 4.5 \\
\hline LU & 1851 & -1318 & 542 & 19.9 & 21.4 & 3.7 & 3.9 \\
\hline LV & 1857 & -1023 & 883 & 21.3 & 22.2 & 4.1 & 4.3 \\
\hline
\end{tabular}

Notes. The windows are identified by a local identifiers as in Dominici et al. (1999).

\section{The catalog}

The catalog was constructed only with those stars with 20 observations or more, observed in at least six different years and at least three times each year. Moreover, we selected only the stars with a time base equal to ten years or more. This filtering ensures a good compromise between the number of observations, the observational epochs, and the time base, giving excellent precision on positions and proper motions. Finally, the stars constituting the GBVOC are spread in a region of $5 \mathrm{~min}$ in right ascension by $14^{\prime}$ in declination around each low-extinction window whose sizes are estimated about $20^{\prime} \times 20^{\prime}$. The final resulting catalog contains 11619 stars covering a total zone of more or less $15.0^{\circ} \times 0.2^{\circ}\left(12\right.$ regions of $\left.5 \mathrm{~min} \times 14^{\prime}\right)$ giving a density of more than 3500 stars by $1^{\circ} \times 1^{\circ}$.

The central coordinates of each low-extinction window are given in Table 1, where we also give some statistics such as the number of stars, the mean internal precision on positions, and proper motions in each window direction.

In a general way, this catalog provides precise and homogeneous positions and proper motions in the ICRF as realized by the Tycho- 2 catalog (Hög et al. 2000). In this way, it represents a very good densification in the optical domain of the ICRF reaching a limiting magnitude around 16.0 mag in the $V$ band, as shown in Fig. 1. This limitation in magnitude means that only a few stars in our catalog belong to the bulge, most of them being foreground stars.

As we can see in Fig. 2, where we present the distribution of the number of observations per star, most of the stars of the GBVOC have a very good observational history. Most stars were observed more than 50 times and the average number of observations per star is higher than 80 in both coordinates. The number of observations in right ascension and declination is not exactly the same as a consequence of independent eliminations in the reduction process, but this is only the case for very few stars.

This relatively very large number of observations ensures the high quality of the resulting positions. On average we have a mean internal precision of about 22 mas in both coordinates, as can be seen in the distribution of the internal precision in both coordinates shown in Fig. 3.

In Fig. 4 we show the internal precision on position as a function of magnitude. In this figure, dark is used for RA and red for Dec. We can note that even at the limit of magnitude the mean internal precision of the GBVOC positions remains

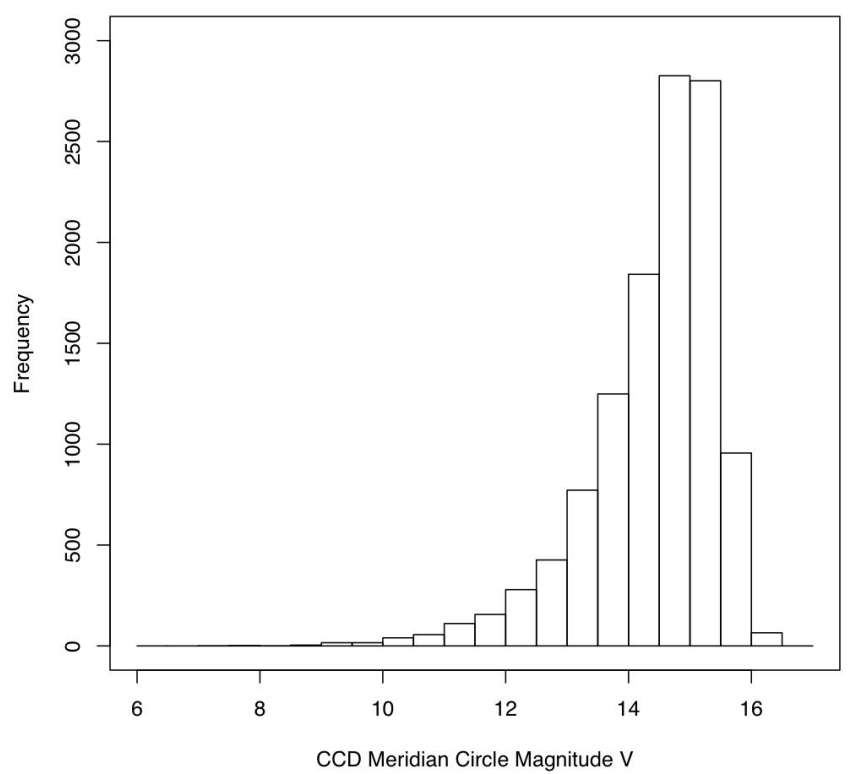

Fig. 1. Distribution of the stars by $V$-band magnitudes in the Galactic Bulge Valinhos Observatory Catalog (GBVOC).
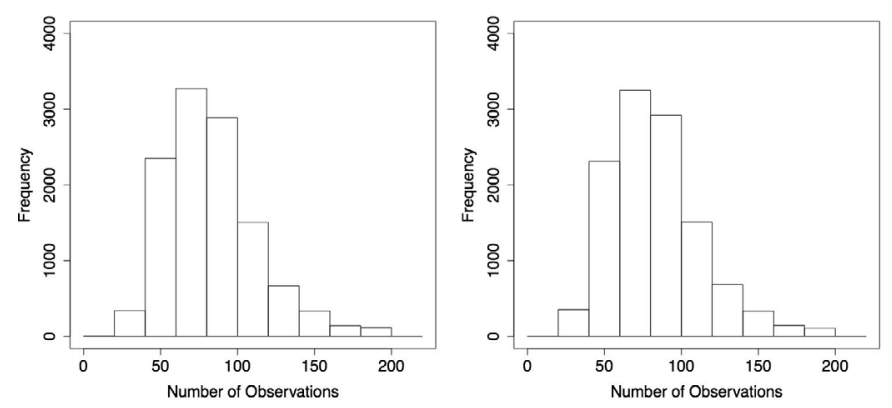

Fig. 2. Distribution of number of observations in right ascension (left panel) and declination (right panel) of the stars composing the GBVOC.
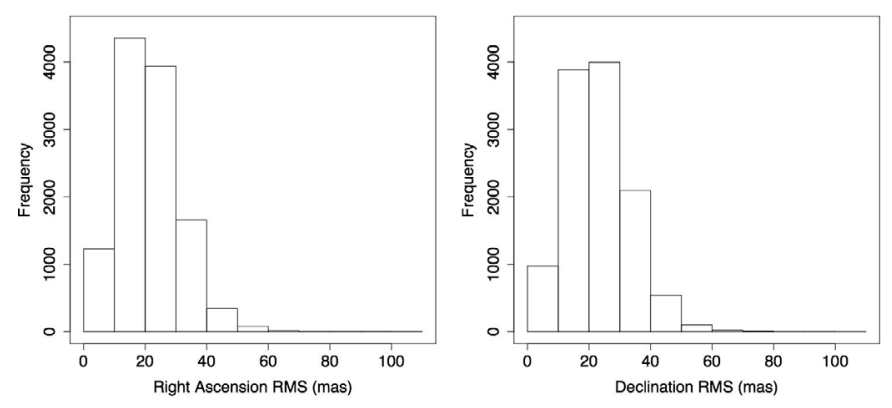

Fig. 3. Distribution of the internal precision achieved (rms) in right ascension (left panel) and declination (right panel).

highly compatible with those given in the best astrometric catalogs. Each point represents the mean of the rms of hundreds of stars. Only the first point is an exception because it results from only a few bright stars. For these stars the images were mostly saturated, which explains their lower quality. The limit of saturation in the Valinhos CCD meridian circle is about 8.5 mag.

The scenario is the same for proper motions. We provide homogeneous and precise proper motions for all stars. Their mean internal precision is about $4.0 \mathrm{mas} / \mathrm{yr}$, which is also compatible with the best astrometric catalogs. Of course, this precision depends on magnitudes and degrades for faint objects. In Fig. 5 we present the distribution of the proper motions of our 


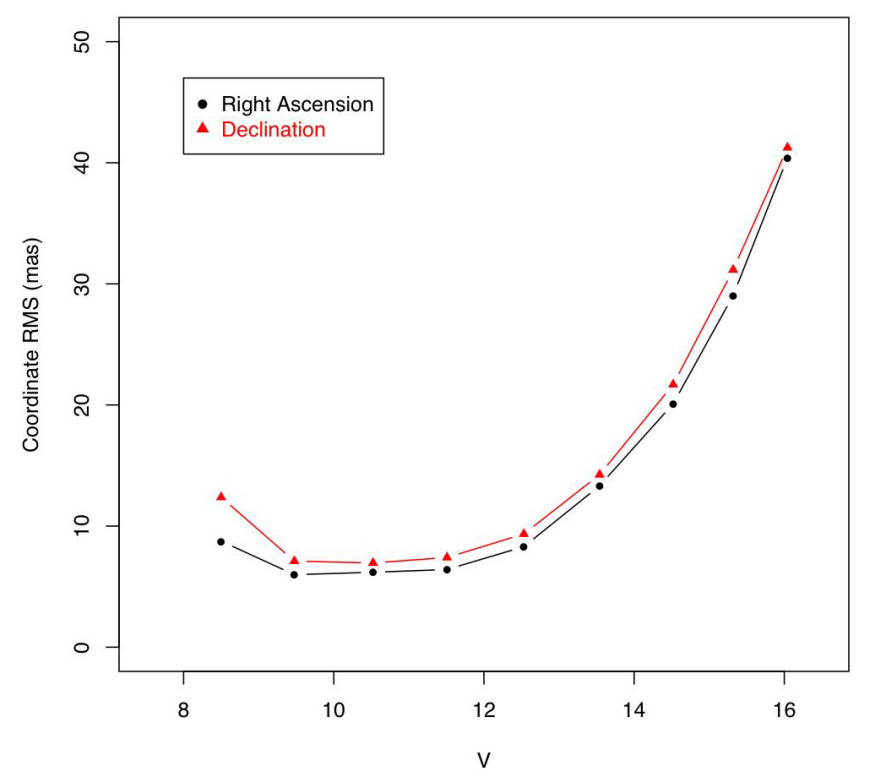

Fig. 4. Mean internal positional precision in right ascension (dark) and declination (red) as function of magnitude.
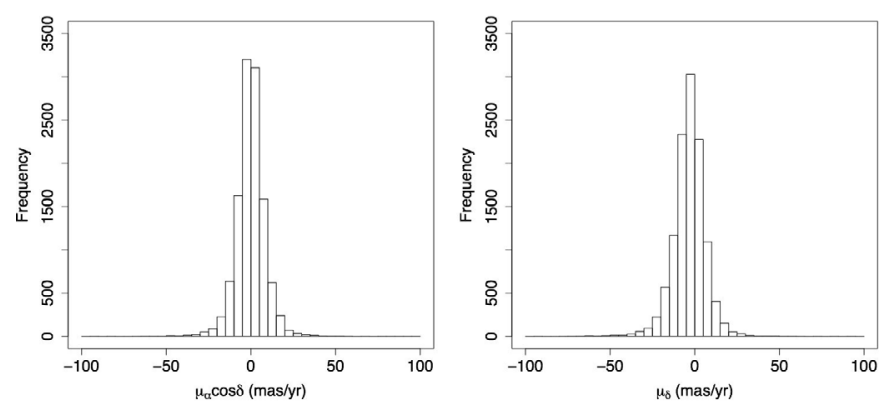

Fig. 5. Distribution of the proper motions in right ascension (left panel) and declination (right panel) in the GBVOC.
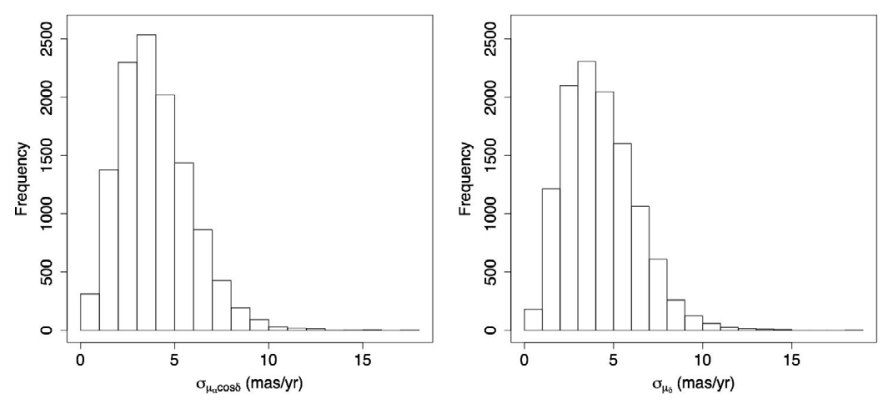

Fig. 6. Distribution of the internal precision achieved (rms) in right ascension (left panel) and declination (right panel) proper motions.

catalog. In Fig. 6 we show the distribution of the internal errors in proper motion. As can be noted here the distribution is very sharp (0-10 mas/yr), reflecting the high homogeneity of our proper motions.

In Fig. 7 we show the variation in the mean internal precision in proper motion as a function of magnitude. In this figure we can see that on average the internal errors in our proper motions in the best interval (until $V \simeq 14.0$ ) are lower than 2 mas/yr. Again, the first point does not follow the general trend because it is the mean of only a few bright stars whose images were frequently saturated. The quality of the positions and proper motions given in the GBVOC associated to the tight control of the

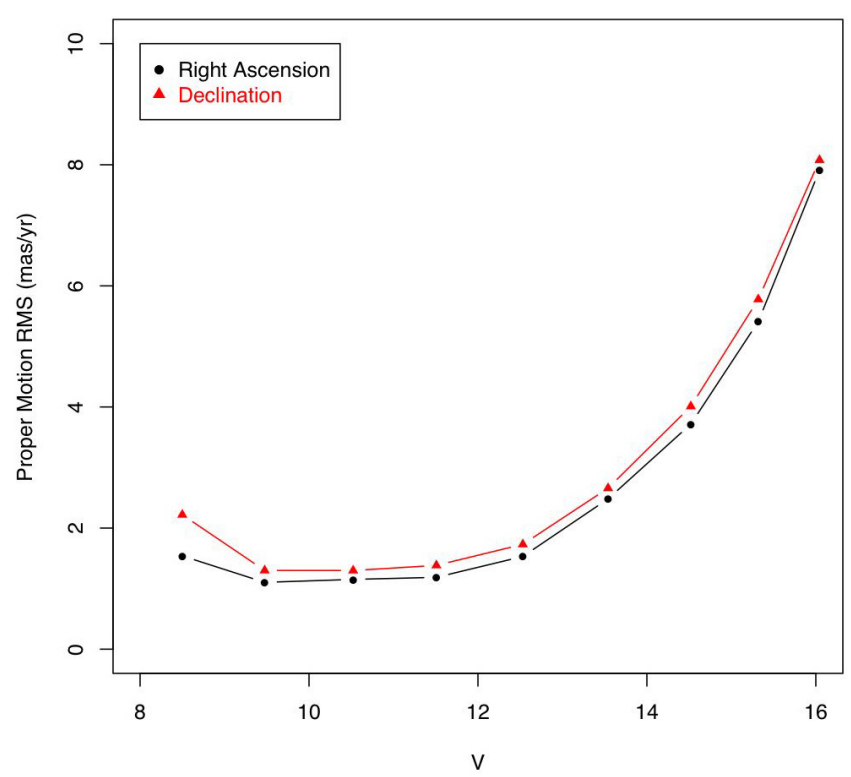

Fig. 7. Mean internal precision in right ascension (dark) and declination (red) proper motion as function of magnitude.

whole process of astrometric reduction transforms this artisanal work into an important extension of the ICRF in a very interesting region of the sky.

Although GBVOC is an astrometric catalog, we used the $J H K$ photometry of the 2MASS for calculating the infrared colors of our stars in order to estimate their effective temperature. We found 2MASS $J H K$ photometry for $98.2 \%$ of the GBVOC stars and we used it to compute their $J-K$ and $H-K$ colors. In their recent publication Worthey \& Lee (2011) present an empirical $U B V R I J H K$ color-temperature calibration for stars. We used this calibration and the $J-K$ and $H-K$ colors to derive the $T_{\text {eff }}$, which corresponds to the stars in our catalog.

We were not able to consider the reddening correction, which corresponds to the stars of GBVOC, because we do not know their distances. But since the stars in our catalog have $V \leq 16 \mathrm{mag}$, they are not so far so that they cannot be members of the Galactic bulge, since they are foreground stars. In any case, GBVOC contains stars that are in fields with low extinction. Nishiyama et al. $(2005,2006,2008)$ determined the ratios of total to selective extinction directly from observations in the optical $V$ band and near-infrared, and obtained $A_{V}: A_{J}: A_{H}: A_{K} \mathrm{~s}=$ 1:0.188:0.108:0.062 for reddened fields near the Galactic center. Considering these extinction ratios, it is not very important to apply a reddening correction to the $J-K$ and $H-K$ colors of the GBVOC stars.

Considering that GBVOC stars are in or close to Galactic disk we restricted our search to part of the Worthey \& Lee (2011) calibration concerning the population I stars with $[\mathrm{Fe} / \mathrm{H}] \geq 0.0$.

To calculate the $T_{\text {eff }}$ of each GBVOC star we calculated first the differences $\Delta(J-K)=(J-K)_{2 \text { MASS }}-(J-K)_{\text {Worthey }}$ and $\Delta(H-K)=(H-K)_{2 \mathrm{MASS}}-(H-K)_{\text {Worthey }}$ for all corresponding colors in the calibration. Then we determined as the effective temperature of a star the $T_{\text {eff }}$ value of the Worthey \& Lee (2011) calibration, which corresponds to the value of $\min \left(\Delta(J-K)^{2}+\right.$ $\left.\Delta(H-K)^{2}\right)$.

We present the distribution of the effective temperatures of the GBVOC stars in our sample in Fig. 8. We note that most of the stars have temperatures lower than $7000 \mathrm{~K}(98.5 \%)$. A large part of them have approximately solar temperatures of 


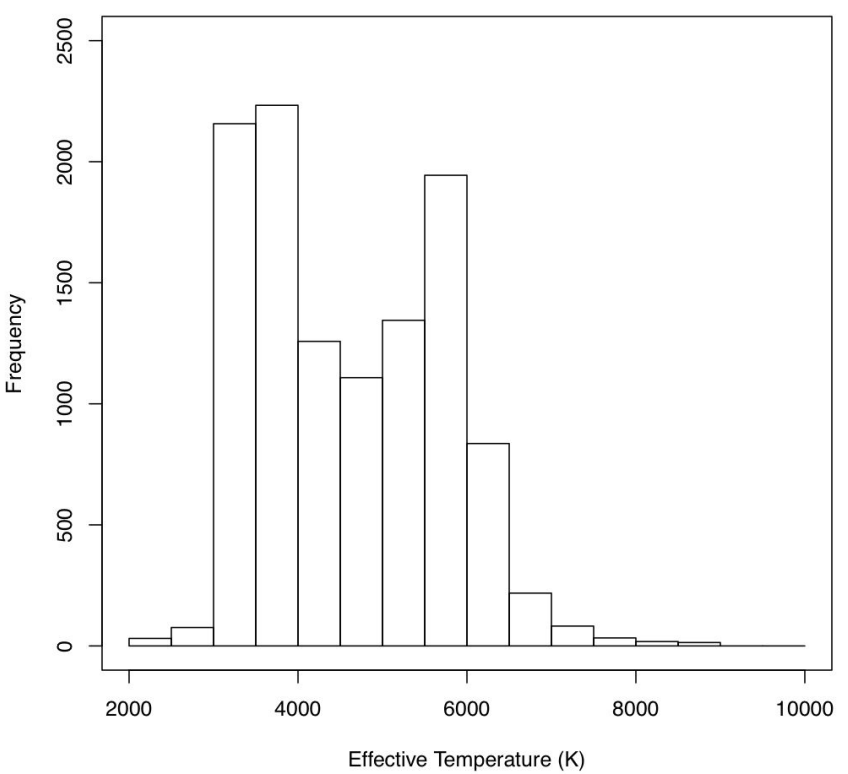

Fig. 8. Distribution of the effective temperature $T_{\text {eff }}$ of the stars of GBVOC.

$T_{\mathrm{eff}} \approx 5800 \pm 500 \mathrm{~K}(28.5 \%)$. Finally, many of the GBVOC stars are cool, with temperatures of $3500 \pm 500 \mathrm{~K}(38.5 \%)$.

The calibration classified $40 \%$ of these cool stars as having at least solar surface gravity $(\log g \geq 4$.0) with a mean magnitude of $11.6 \pm 0.9 \mathrm{mag}$, while an additional $32 \%$ of the stars in the same group of cool stars were classified as having $\log g \leq 1.0$, and their calculated mean magnitude is $10.3 \pm 1.0 \mathrm{mag}$.

Cool stars with $\log g \leq 1.0$ do not only show a tendency to be brighter than cool ones with $\log g \geq 4.0$, but they also have a tendency to be cooler, since their average effective temperature is $3420 \pm 200 \mathrm{~K}$, while stars with $\log g \geq 4.0$ have an average of $3620 \pm 250 \mathrm{~K}$. This means that cool stars classified with a low surface gravity of $\log g \leq 1.0$ are brighter and redder that the corresponding ones with at least solar surface gravity. Although the $\log g$ estimation derived by our method is not reliable, this finding does not contradict to stellar theories.

We compared our $T_{\text {eff }}$ estimation with values taken from the literature for all GBVOC stars brighter than $V=10$ mag. The precision is about $70 \mathrm{~K}$, but the accuracy is disturbed by reddening, giving about $500 \mathrm{~K}$ systematically lower values.

Finally, in Fig. 9 and Table 2 we conclude this overview of the Galactic Bulge Valinhos Observatory Catalog by showing the distribution of the mean epochs and an extract of the catalog. The mean epoch of our catalog for both coordinates is around 2002. In a few cases, the mean epoch is not exactly the same in both coordinates as a consequence of independent elimination of measurements. Figure 9 illustrates the distribution of the mean epoch in both coordinates.

In Table 2 we present an extract of the GBVOC where we provide all essential information to use it properly. The meaning of each column is in general clear, except the "ident" column which gives a local identifier and the " $J$ ", " $H$ ", and " $K$ " columns that give the 2MASS magnitudes (Cutri et al. 2003). The capital letter " $T$ " after the 2MASS identifier indicates that this star is also presented in the Tycho 2 catalog. The $V$ magnitude given in this table corresponds to the magnitude observed with the Valinhos CCD meridian circle that is very close to the Tycho $V$ magnitude (Dominici et al. 1999). The positions are given to the mean epoch of observations.
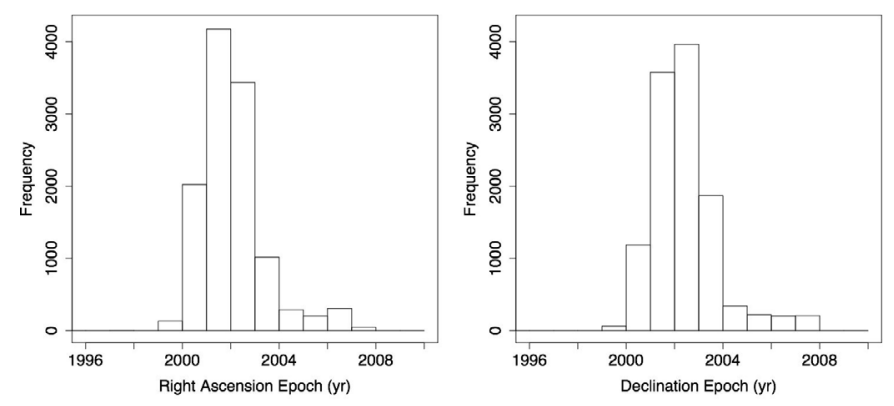

Fig. 9. Mean epoch distribution of the right ascension (left panel) and declination (right panel) in the GBVOC.

The catalog is divided by windows, as in Table 1 in which the stars are listed in order of ascending right ascension.

\section{Analysis and external precision}

Despite the very good internal precision of our astrometric measurements, it is important to discuss and evaluate the quality of our results by comparing them with other astrometric catalogs, as independently as possible. At the present time, there are a few very good astrometric catalogs such as Tycho-2 (Hög et al. 2000), PPMX (Röser et al. 2008) and UCAC3 (Zacharias et al. 2010) for instance. Each of these catalogs attempts to answer some of the basic requirements for the realization of a reference system, but despite their good quality, no one fills it completely. Soon, we will have the UCAC4 (Zacharias 2011, priv. comm.), which has not been published yet, but is used here as requested by the referee of this paper.

For example, Tycho-2 gives very precise positions and proper motions but is very limited in magnitude. The UCAC3 is deep in magnitude, relatively dense but the quality of its positions and proper motions is not as high as one would prefer, and much lower than those of Tycho-2. Moreover, now, this catalog is practically outdated (Zacharias 2011, priv. comm.). The PPMX, on the other hand, is much denser than Tycho- 2 but much less than UCAC3 and UCAC4. Its position and proper motion system is very accurate for its more precise components, but the catalog contains relatively few faint stars.

In fact, such comparisons of catalogs only crudely estimate our uncertainties. The difficulty comes, for instance, from the fact that the internal precisions are not necessarily the best representation of their quality. Even so, these comparisons are important and allow us to obtain, at least, an estimate of the upper limit of the errors in our catalog.

\subsection{Tycho-2 comparison}

The Tycho- 2 catalog (Hög et al. 2000) has about 2.5 million stars that are brighter than $V \simeq 12$ mag, nearly complete until $V \simeq$ $11 \mathrm{mag}$. This catalog results of the combination of various other catalogs and the astrometric observations of the sky mapper of the Hipparcos satellite. Their positions and proper motions are very precise, reaching $\simeq 60$ mas and $\simeq 2.5 \mathrm{mas} / \mathrm{yr}$.

This precision makes it an interesting reference for evaluate the external quality of the Galactic Bulge Valinhos Observatory Catalog. Of course, as we took this catalog as a reference in our reduction, our results depend on the Tycho- 2 positions and proper motions. However, owing to the global strategy of reduction applied here, this dependence is very weak, concerning 


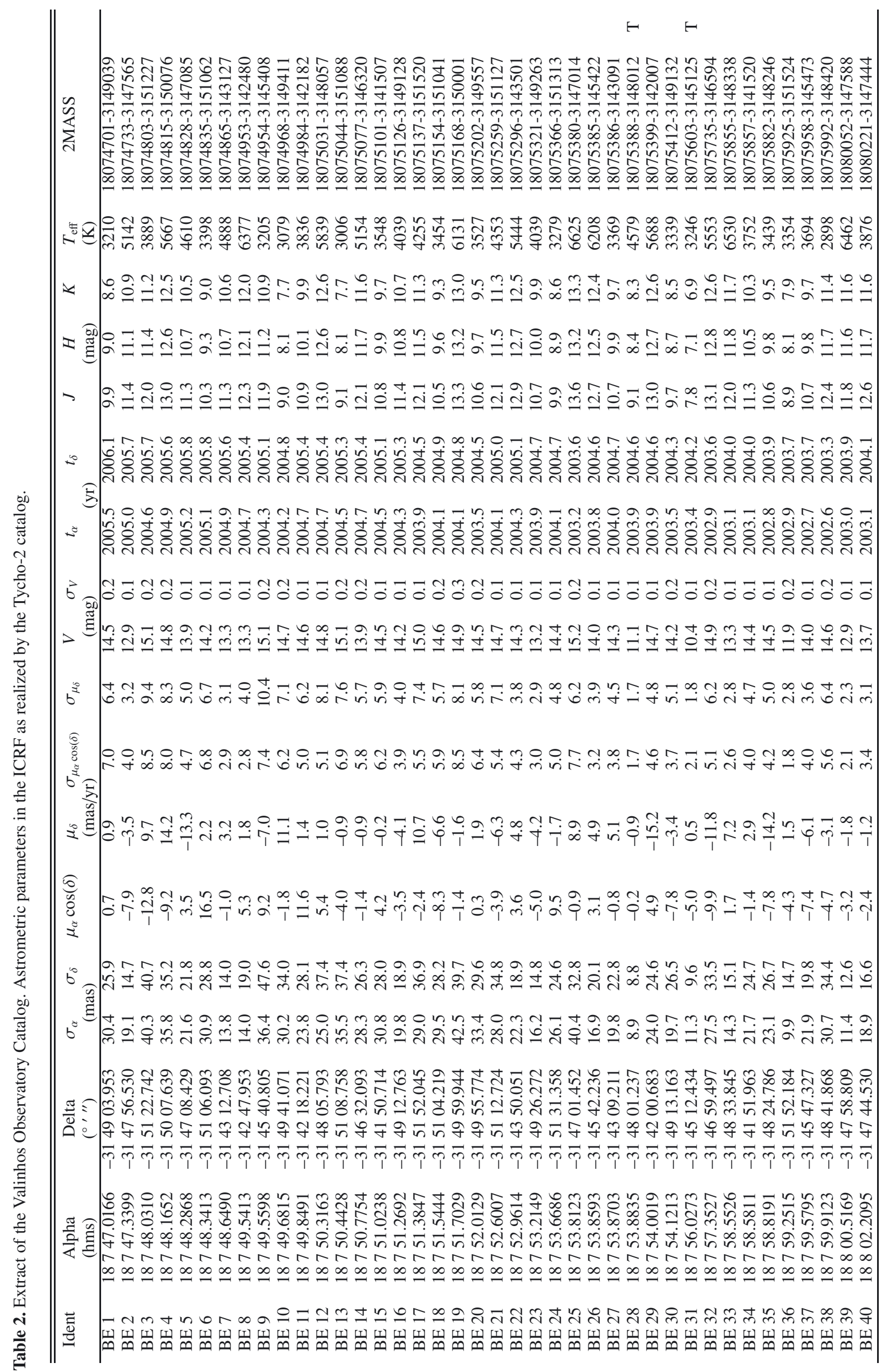



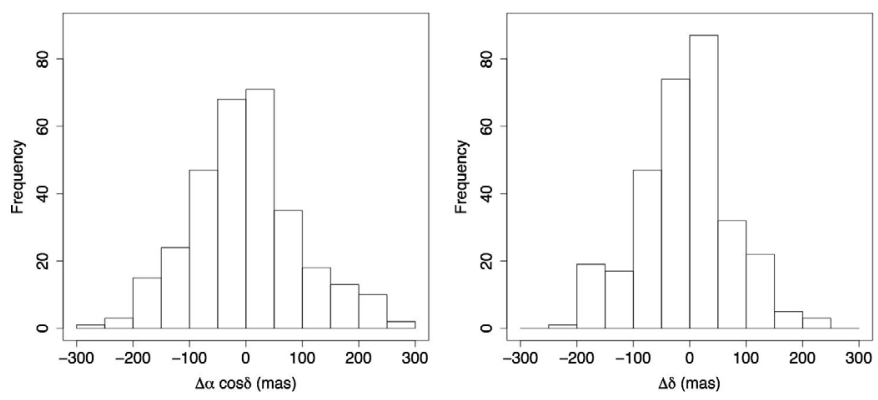

Fig. 10. Distribution of the differences GBVOC - Tycho 2 in right ascension (left panel) and declination (right panel).

only the orientation of the axes (Ducourant \& Rapaport 1991; Benevides-Soares \& Teixeira 1992; Teixeira et al. 1992). Even as the reference of the GBVOC reduction, Tycho- 2 can still give us a reliable estimate of the quality of our position and proper motion measurements.

First of all, we compared the GBVOC positions with those from Tycho-2 at the epoch 2000.0 (published epoch) in Fig. 10. We found 271 Tycho-2 stars in the GBVOC. In fact, this number should be higher, but because our purpose is to evaluate the GBVOC external precision, we selected here stars for which there was no doubt of the cross identification. Of course, some stars may have escaped from our filter, but certainly in an insignificant proportion. This is valid for the other comparisons.

As a result, we obtain a mean difference of -11.8 mas in right ascension and -6.7 mas in declination, with dispersions of 76.0 mas and 72.3 mas, respectively. These values show good agreement between our positions and those from the Tycho-2 catalog. The distribution of the mean differences (GBVOC Tycho-2) shown in Fig. 10 indicate that there are no significant systematic effects in our position system. The agreement, estimated by the dispersion, which can be considered good, could be even better if many stars were not in the magnitude limit of the Tycho- 2 catalog, where its quality is not very good (Hög et al. 2000). Moreover, we have a few bright stars here whose images were saturated in the CCD meridian circle.

If we take the mean errors on the Tycho- 2 right ascension and declination for these common stars into account, 56.7 mas and 69.5 mas, we can estimate the external precision of our positions to 50.6 mas and 19.9 mas in right ascension and declination. This difference between the estimated external precision in right ascension, and declination comes from the reference cata$\log$, Tycho-2, where the mean errors of the two coordinates are significantly different. Even so, we can say from this comparison that the external precision in the GBVOC positions are, on average, better than 50 mas.

In the case of the proper motions, the mean difference are $-1.2 \mathrm{mas} / \mathrm{yr}$ and $0.2 \mathrm{mas} / \mathrm{yr}$ with dispersions of $4.1 \mathrm{mas} / \mathrm{yr}$ and $5.1 \mathrm{mas} / \mathrm{yr}$ in right ascension and declination, respectively. These values suggest very good agreement between the proper motions of the two catalogs. If we also consider the Tycho- 2 proper motion errors, we conclude that the mean external precision of our proper motion is $3.9 \mathrm{mas} / \mathrm{yr}$, hence very near of the internal mean precision.

Figure 11 gives the comparison between our proper motions and those from the Tycho-2 catalog for 271 common stars. This figure leaves no doubt about the good agreement between these proper motions. The good distribution along the diagonal confirms the absence of systematic effects between these two catalogs in the considered region. Unfortunately, the Tycho-2
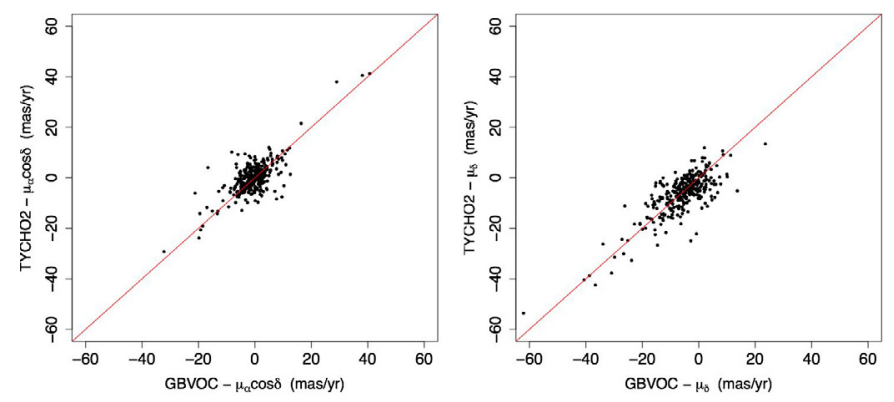

Fig. 11. Proper motion comparison with Tycho 2. Right ascension proper motion (left panel) and declination proper motion (right panel).

limiting magnitude restricts this comparison to the brighter stars where the meridian measurements are better, as in Fig. 7.

To summarize, for the brighter segment of our catalog we have in average, an external precision estimated from comparison with Tycho- 2 catalog better than 50 mas on position and $4 \mathrm{mas} / \mathrm{yr}$ on proper motion.

\subsection{UCAC4 comparison}

To estimate the external precision of our catalog for the fainter stars, initially we used the UCAC3 catalog (Zacharias et al. 2010) and then, following the suggestion of the referee of this paper, we worked with the new version of this series, the UCAC4 (Zacharias 2011, priv. comm.). We therefore took advantage of a beta version of the UCAC4 catalog kindly provided by Dr. N. Zacharias (2011, priv. comm.). In the very near future, the UCAC4 will be one of the most important astrometric catalogs with positions and proper motions better than those from UCAC3 (15-100 mas and 1-10 mas/yr). The UCAC4 like its predecessor will contain more than 100 million objects covering the whole sky. Its astrometric parameters result from the recent astrographic observations of the two hemispheres combined with those from many earlier catalogs.

Certanly, this catalog will be a very important extension to the ICRF mainly thanks to its amount of stars and limiting magnitude $(R \simeq 16)$. Its internal astrometric precision is better than that of UCAC3, and it probably presents a degradation in the fainter segment where, in general, the measured proper motions are only based on a few points, frequently on only two to four points (observational epochs). Nevertheless, we took it as an external reference to evaluate the GBVOC position and proper motion quality mainly for the faint stars, because almost all of our stars are present in the UCAC4 catalog.

In Fig. 12 we can see the distribution of the differences, at the epoch 2000.0 (published epoch), in right ascension (left) and declination (right) for about 8000 common stars. We were very restrictive in the selection of common stars working only with those separated by at most 200 mas. In this case we obtain a mean difference in right ascension and declination of 45.6 mas and -17.6 mas with dispersions of 60.4 mas and 43.2 mas. Taking the mean errors for these UCAC4 positions into account (22.9 mas in right ascension and 22.7 mas in declination), we can estimate the external precision for the GBVOC in 55.9 mas in right ascension and 36.8 mas in declination. The mean difference in right ascension suggests a significant systematic deviation in this coordinate between both catalogs. This deviation can also be seen in Fig. 12. Only based on this comparison we cannot decide which catalog is responsible for this effect. We also 

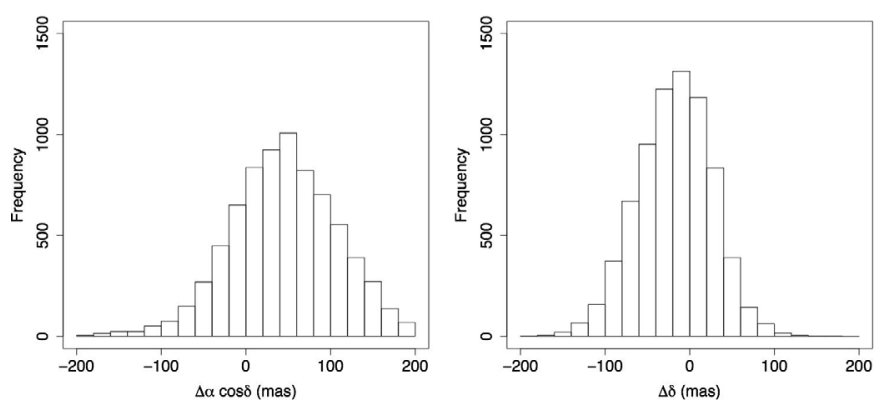

Fig. 12. Distribution of the differences GBVOC - UCAC4 in right ascension (left panel) and declination (right panel).
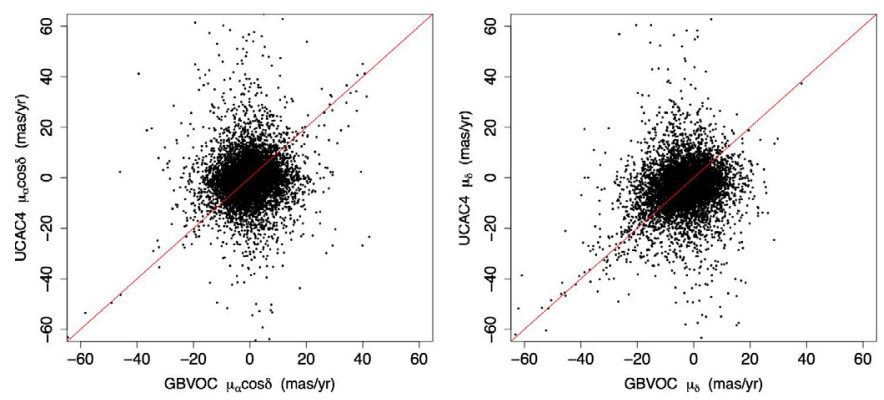

Fig. 13. Proper motion comparison with UCAC4. Right ascension proper motion (left panel) and declination proper motion (right panel).

note that the external errors based on the UCAC4 positions are twice the internal ones.

For the proper motions the mean differences are $0.6 \mathrm{mas} / \mathrm{yr}$ and $0.5 \mathrm{mas} / \mathrm{yr}$ with a dispersion of $10.1 \mathrm{mas} / \mathrm{yr}$ and $10.0 \mathrm{mas} / \mathrm{yr}$ in right ascension and declination respectively. The dispersions are greater than what we observe from the Tycho- 2 comparison, and it is probably a consequence of our working with fainter stars for which the quality of astrometric measurements is lower. When taking the UCAC4 mean internal errors into account for these stars (4.2 mas/yr and $4.1 \mathrm{mas} / \mathrm{yr}$ ), the estimated external mean errors for the GBVOC proper motions are $9.2 \mathrm{mas} / \mathrm{yr}$ and $9.1 \mathrm{mas} / \mathrm{yr}$. These values are very high and far from our internal estimate. The reason for that can be attributed to the relatively large dispersion of the differences in the case of the faint stars as showed in the following figures and discussion.

In Fig. 13 we illustrate the comparison between the UCAC4 and GBVOC proper motions. The behavior seen in these figures is quite unexpected. We expected a much larger dispersion than for Tycho-2 but still with a diagonal trend and without the vertical structure that we can see.

We note in these figures that, while the UCAC4 proper motions spread and reachs high values (we even have some points outside the graphic), in general, GBVOC remains concentrated between -20 mas/yr to $20 \mathrm{mas} / \mathrm{yr}$. We cannot be sure of the origin of this behavior, but it is clear that the UCAC4 proper motions are much more dispersed than ours.

Although our aim in this paper is not to test UCAC4, the unexpected behavior seen in Fig. 13 forces us to conduct a more detailed study to better understand what is happening. First of all, in our comparison with the Tycho- 2 catalog, we are led to believe that this difference comes from the faint stars, since our agreement with Tycho-2 (magnitude limit about $V \simeq 12.0$ ) is very good. To confirm that, we present in Fig. 14 the same comparisons but highlighting in orange the stars brighter than 12.0. As we can see, our proper motions are in good agreement with those from UCAC4 for the bright stars, and as a consequence
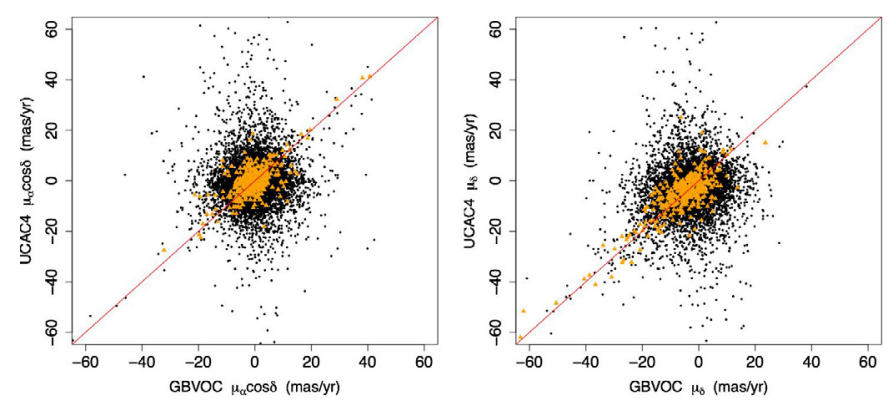

Fig. 14. Proper motion comparison with UCAC4. Right ascension proper motion (left panel) and declination proper motion (right panel) black dots $=$ all stars, red dots $=$ stars brighter than $V \simeq 12$.
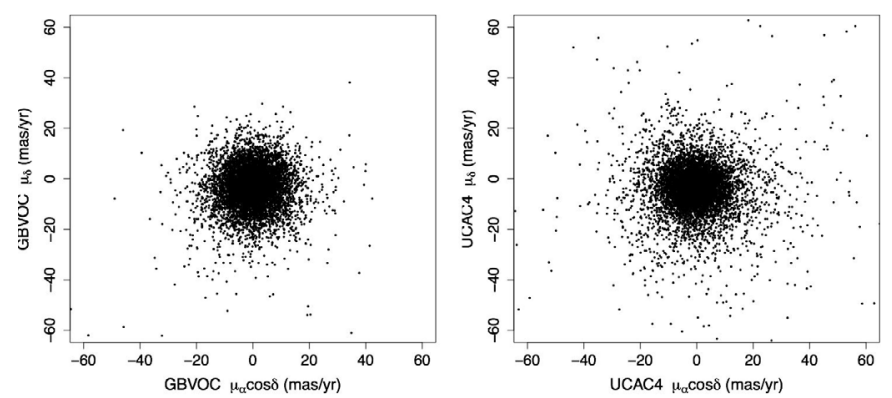

Fig. 15. Vector point diagram for Valinhos proper motions (left panel) and for UCAC4 proper motions (right panel).

the vertical structures seen in Figs. 13 are due to the faint stars. Unfortunately, this figure does not reveal the origin of the problem.

In Fig. 15 we plotted the proper motions in declination as a function of the proper motions in right ascension for both catalogs, GBVOC in the left panel and UCAC4 in the right. As we can see, the results obtained with the GBVOC is noticeably less spread than those with UCAC4. It is very difficult to explain the origin of these differences. We think that, since most stars are faint, the expected behavior is a less dispersed figure, as we see in the case of GBVOC proper motions. The large dispersion in UCAC4 could come from some bad proper motion determinations as a consequence of the automation in dealing with a huge amount of data and also from the higher density of stars in the direction of low-extinction windows.

The following diagrams give the GBVOC (left) and UCAC4 (right) proper motions as a function of the UCAC4 magnitude. In these cases we expected greater dispersion for the fainter stars as consequence of the lower precision. In other words, as the proper motion errors is larger for the fainter stars the dispersion should increase for higher magnitudes. It is easy to see this behavior in both catalogs. However, we also can see an unexpected concentration of larger proper motions from UCAC4 around magnitude 14 . The more likely in this case would be to have this concentration around the brighter stars. So, apparently the structure that we see in the comparison GBVOC $\times$ UCAC4 (Fig. 13) comes from UCAC4.

In either case, we cannot say for sure what the problem with the larger differences is. Since our goal in this paper is not to discuss the beta version of the UCAC4 catalog, we leave a more detailed analysis to more specific work in this direction.

Considering the behavior seen in these figures, only the comparison with UCAC4 is not sufficient for estimating the external qualities of our positions and proper motions. Moreover, 

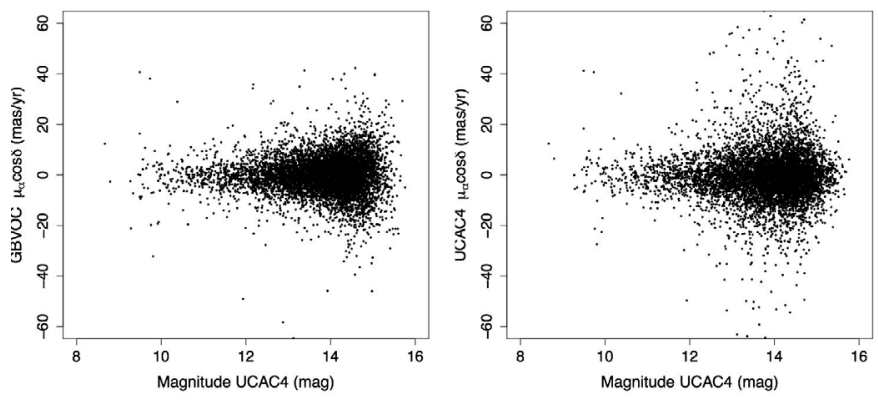

Fig. 16. Right ascension proper motion as a function of the UCAC4 magnitude: GBVOC (left panel) and UCAC4 (right panel).
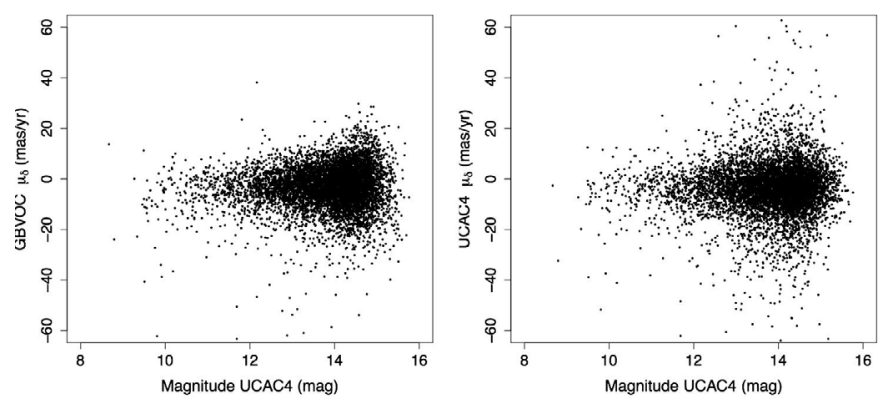

Fig. 17. Declination proper motion as a function of the UCAC4 magnitude: GBVOC (left panel) and UCAC4 (right panel).

the results of this comparison must be seen as encouraging independent measurement of the same astrometric quantities.

\subsection{PPMX comparison}

Owing to the observed and unexpected structure in Fig. 13, we decided to consider another catalog for comparison. An other possibility for estimating the GBVOC external precision is the recent PPMX catalog (Röser et al. 2008). The PPM-Extended catalog, PPMX, is another important extension of the ICRF in the optical domain. It contains about 20 million stars up to $V \simeq 15-16 \mathrm{mag}$, with a typical precision in proper motion of 1-2 mas/yr (Röser et al. 2008). Of course, this catalog is less dense than UCAC4, and the number of common stars is much smaller.

As in Sect. 5.2 we compared our positions and proper motions with those from PPMX catalog for 950 common stars. The obtained mean differences in right ascension and declination at the PPMX published epoch (2000.0) are 30.2 mas and -20.4 mas with dispersions of 58.7 mas and 55.4 mas. Taking the PPMX mean internal errors into account for these stars (34.7 mas and 35.2 mas), we estimated the GBVOC external mean errors in 47.3 mas and 42.8 mas in right ascension and declination. This comparison may be seen in Fig. 18.

The mean differences in proper motions in right ascension and declination are $-1.8 \mathrm{mas} / \mathrm{yr}$ and $2.9 \mathrm{mas} / \mathrm{yr}$ with dispersions of $11.4 \mathrm{mas} / \mathrm{yr}$ and $12.3 \mathrm{mas} / \mathrm{yr}$. Considering that the mean of the internal PPMX errors in proper motion in both coordinates is $9.8 \mathrm{mas} / \mathrm{yr}$ for these common stars, we can estimate the GBVOC external mean proper motion error as about $6.5 \mathrm{mas} / \mathrm{yr}$, not much larger than the internal proper motion error 4.0 mas/yr. The mean differences in position and proper motion are lower than the mean PPMX internal errors, suggesting no significant systematic deviation between both catalogs.
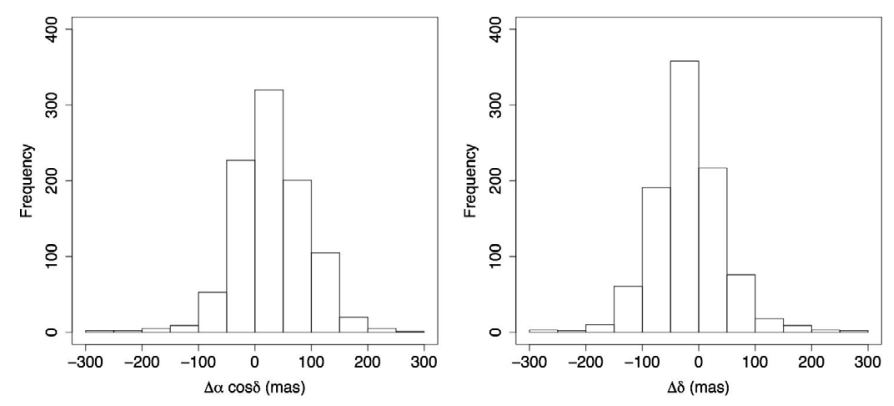

Fig. 18. Distribution of the differences GBVOC - PPMX in right ascension (left panel) and declination (right panel).
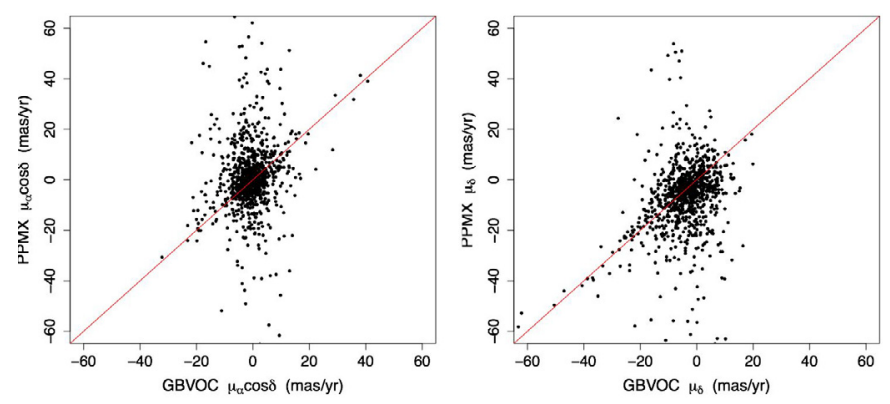

Fig. 19. Proper motion comparison with PPMX. Right ascension proper motion (left panel) and declination proper motion (right panel).
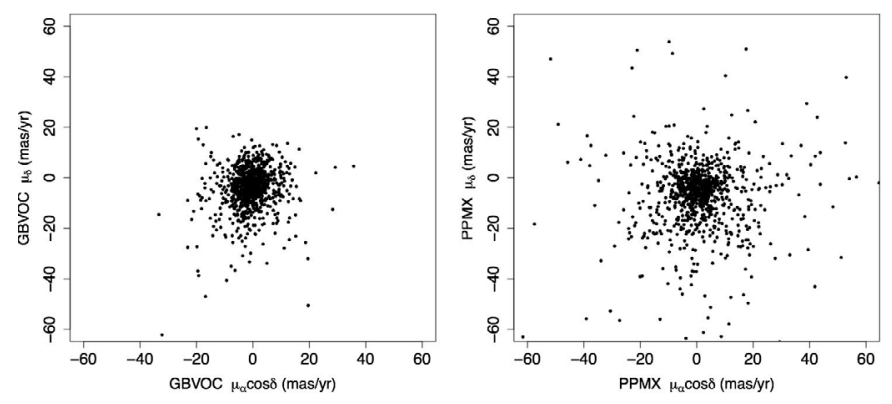

Fig. 20. Vector point diagram for Valinhos proper motions (left panel) and for PPMX proper motions (right panel).

In Fig. 19 we illustrate the results of the comparison between the PPMX and GBVOC proper motions. We do not have the clear diagonal trend one could expect. More or less as in the previous case (UCAC4), we can see a dispersion in the PPMX proper motions when the GBVOC values stay between -20 and $20 \mathrm{mas} / \mathrm{yr}$. The observed proper motion dispersion is lower than that of the UCAC4 comparison probably because we are working here with few common stars.

In Fig. 20 we plot the declination proper motion as a function of the right ascension proper motion for both catalogs. This suggests that the pattern seen in Fig. 19 comes from the differences in the measured proper motions.

Since the PPMX catalog is composed of three components (flags "S", "H", and "O", Röser et al. 2008) to better estimate our external precision, we chose to compare our positions and proper motions only to the best stars from the PPMX. We therefore constructed others graphs (Figs. 21 and 22) but only with those stars whose PPMX internal position and proper motion precision is highest, i.e., for the stars with a flag "S" (survey) and " $H$ " (highest precision). We found 620 common stars that give mean differences in right ascension and declination of 21.4 mas and -18.0 mas with dispersion of 50.6 mas and 47.3 mas, resulting 

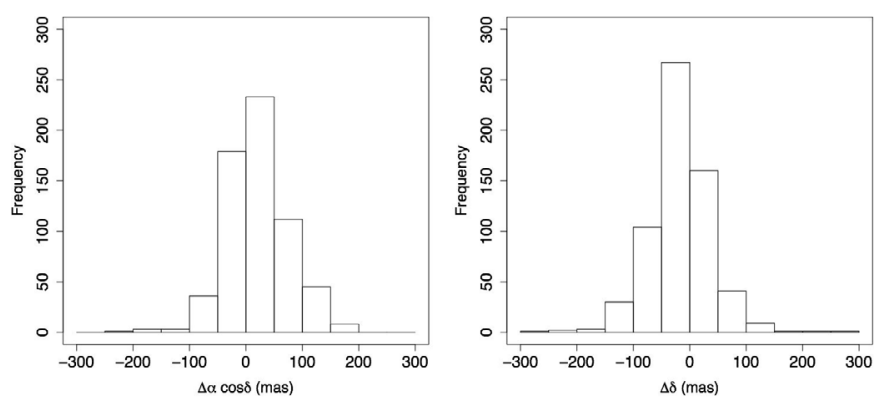

Fig. 21. Distribution of the differences GBVOC - PPMX (flags "S" and "H") in right ascension (left panel) and declination (right panel).
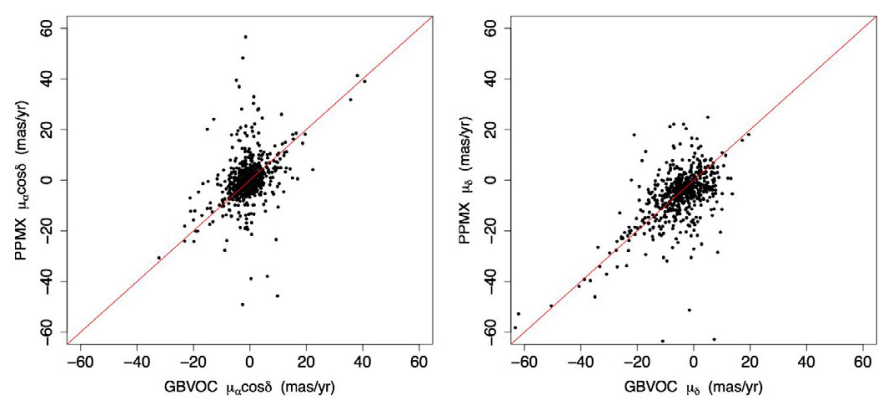

Fig. 22. Proper motion comparison with PPMX (flags "S" and "H"). Right ascension proper motion (left panel) and declination proper motion (right panel).

in an external error estimate for our positions of 40.8 mas in right ascension and 36.5 mas in declination.

For the proper motion, we obtained mean differences of $-0.7 \mathrm{mas} / \mathrm{yr}$ and $0.9 \mathrm{mas} / \mathrm{yr}$ in right ascension and declination proper motions with dispersion, respectively, of $6.7 \mathrm{mas} / \mathrm{yr}$ and $7.5 \mathrm{mas} / \mathrm{yr}$. Here there is no systematic effect. As we can see, the perpendicular structure to the abscissas axis almost disappeared, suggesting that the structure in Fig. 19 comes from the PPMX stars with lower accuracy. Considering that the mean internal errors for this segment of the PPMX catalog are 5.4 mas/yr and $5.5 \mathrm{mas} / \mathrm{yr}$ in proper motion in right ascension and declination, we can estimate the mean external precision of our proper motions as about $4.0 \mathrm{mas} / \mathrm{yr}$ and $5.1 \mathrm{mas} / \mathrm{yr}$, very close to the internal mean precision of GBVOC.

With this selection of PPMX stars ("S" and " $\mathrm{H}$ " stars), we could only compare the stars that are brighter than $V \simeq 14.0$ mag. The results in this case are not very different from those with the complete PPMX. From this part of the PPMX catalog, we can say that our positions are better than 40.8 mas, and the proper motions are better than 5.1 mas/yr.

\section{Conclusions}

We present an homogeneous and high-precision astrometric catalog that realizes the ICRF in the optical domain in the region around the lower extinction windows of the Galactic bulge. This catalog can be especially useful as a secondary reference frame and for kinematic studies in these regions.

For an external evaluation of the quality of our catalog, we considered three of the main astrometric catalogs. First, we compared the GBVOC positions and proper motions with those from the Tycho- 2 catalog. In this case we could estimate the mean external precision of our positions as better than 50 mas. For the proper motions, our estimation is of $3.9 \mathrm{mas} / \mathrm{yr}$, which is fully compatible with our internal estimates.

To test the fainter stars, we compared our results with the positions and proper motions from the UCAC4 and PPMX catalogs. From UCAC4 we could estimate a positional precision of about in 46.0 mas and $9.1 \mathrm{mas} / \mathrm{yr}$ in proper motion. Using the PPMX catalog we obtained 35.0 mas in position and $6.5 \mathrm{mas} / \mathrm{yr}$ in proper motion. On the other hand, taking the best of the PPMX catalog we obtain a mean positional external precision around 39.0 mas and in proper motion about 4.6 mas/yr. Eliminating the extreme cases from these comparisons we can estimate our external precision as better than 42.0 mas for the positions and $5.5 \mathrm{mas} / \mathrm{yr}$ for the proper motions.

Given this, we are sure that we are offering the community a good and homogeneous catalog that can be very useful for works on the Galactic bulge. At the same time, our comparison could reveal inconsistencies in some of the most important astrometric catalogs emphasizing the importance of artisanal astrometric works.

Finally, we again stress that the quality of the positions and proper motions in the Galactic Bulge Valinhos Observatory Catalog associated to the tight control of the whole process of astrometric reduction thanks to using relatively few stars transforms this work into an important extension of the ICRF in a very interesting region of the sky.

Acknowledgements. We would like to thank the Brazilian Organism FAPESP, CAPES, and CNPq and the French organizations COFECUB and CNRS. We are also grateful to the referee N. Zacharias for his comments, corrections, and suggestions and for the beta version of UCAC4.

\section{References}

Arias, E. F., Charlot, P., Feissel, M., \& Lestrade, J. F. 1995, A\&A, 303, 604 Baade, W. 1951, Publ. Obs. Univ. Mich., 10, 7

Benevides-Soares, P., \& Teixeira, R. 1992, A\&A, 253, 307

Blanco, V. M. 1988, AJ, 95, 1400

Blanco, V. M., \& Terndrup, D. M. 1989, AJ, 98, 843

Capitaine, N., Guinot, B., \& McCarthy, D. D. 2000, A\&A, 355, 398

Cutri, R. M., Skrustskie, M. F., Van Dyk, S., et al. 2003, 2MASS all Sky Catalog of Point Sources (Pasadena: IPAC/Caltech)

Dominici, T., Teixeira, R., Horvath, J., Medina Tanco, G., \& Benevides-Soares, P. 1999, A\&A, 136, 261

Ducourant, C., \& Rapaport, M. 1991, A\&A, 241, 303

Ducourant, C., Le Campion, J. F., Rapaport, M., et al. 2006, A\&A, 448, 1235

Eichhorn, H. 1960, Astron. Narchr., 285, 233

ESA 1997, HIPPARCOS and Tycho catalogue, ESA SP, 1200

Feissel, M., \& Mignard, F. 1998, A\&A, 331, L33

Fey, A. L., Gordon, D., \& Jacobs, C. S. 2010, IERS/IVS Working Group, IERS Technical Note, 35

Fricke, W. 1985, CeMec, 36, 207

Hög, E., Fabricius, C., Makarov, V., et al. 2000, A\&A, 355, L27

Ma, C., Arias, F. E., Eubanks, T. M., et al. 1998, AJ, 116, 516

Nishiyama, S., Nagata, T., Baba, D., et al. 2005, ApJ, 621, L105

Nishiyama, S., Nagata, T., Kusakabe, N., et al. 2006, ApJ, 638, 839

Nishiyama, S., Nagata, T., Tamura, M., et al. 2008, ApJ, 680, 1174

Platais, I., Girard, M. T., Kozhurina-Platais, V., et al. 1998, AJ, 116, 2556

Roëser, S., Schilbach, E., Schwan, H., et al. 2008, A\&A, 488, 491

Roëser, S., Demkeitner, M., Schilbach, E., et al. 2010, AJ, 139, 2440

Seidelmann, P. K., \& Kovalesvsky, J. 2002, A\&A, 392, 341

Teixeira, R., Requieme, Y., Benevides-Soares, P., \& Rapaport, M. 1992, A\&A, 264, 307

Teixeira, R., Camargo, J. I. B., Benevides-Soares, P., \& Requieme, Y. 1998, A\&A, 333, 1107

Teixeira, R., Ducourant, C., Sartori, M. J., et al. 2000, A\&A, 361, 1143

Viateau, B., Réquième, Y., Le Campion, J. F., et al. 1999, A\&AS, 134, 173

Worthey, G., \& Lee, H. 2011, ApJS, 193, 1

Zacharias, N., Urban, S., Zacharias, M., et al. 2004, AJ, 127, 3043

Zacharias, N., Finch, C., Girard, T., et al. 2010, AJ, 139, 2184 